\title{
Application of exp-function method for non-linear evolution equations to the periodic and soliton solutions
}

\author{
H. Goodarzian, E. Ekrami and A. Azadi \\ Islamic Azad University, Neka Branch, Neka, Iran \\ hamed_goodarzian@yahoo.com
}

\begin{abstract}
In this paper the kaup-kupershmidt, (2+1)-dimensional potential Kadomtsev-Petviashvili (shortly PKP) equations are presented and the exp-function method is employed to compute an approximation to the solution of non-linear differential equations governing on the problem. It has been attempted to show the capabilities and wide-range applications of the exp-function method. This method can be used as an alternative to obtain analytic and approximate solution of different types of differential equations applied in engineering mathematics.
\end{abstract}

Keywords: Exp-function method, kaup-kupershmidt equation, non-linear equations, Kadomtsev-Petviashvili.

Introduction

Non-linear phenomena play important roles in applied mathematics, physics and also in engineering problems in which each parameter varies depending on different factors. Solving non-linear equations may guide authors to know the described process deeply and sometimes leads them to know some facts that are not simply understood through common observations. Moreover, obtaining exact solutions for these problems is a great purpose that has been quite untouched. However, in recent years, analytical solution has considerably been developed to be used for non-linear partial equations (Burden \& Faires, 1993; Kaya \& El-Sayed, 2003). In recently He (2000a,b; 2004a,b; 2005; 2006a,b; 2007) introduced some new method such as variation iteration method (VIM), homotopy perturbation method (HPM) and exp-function method to solve these equations. Expfunction method is very strong for solving high nonlinearity of non-linear equations. Other authors such as Zhu (2007a, b) and Zhang (2007) were working in this field.

\section{Basic idea of exp-function method}

The basic idea of the Exp-function was proposed in He's monograph (He, 2007). Some illustrative examples were given in He (2006b; 2007) and Zhu (2007a, b) showed that this method is very effective to search various solitary and periodic solutions of non-linear equations. Zhang (2007) applied the method to some differential equations with variable coefficient.

We first consider non-linear equation of form

$N\left(U, U_{t}, U_{x}, U_{x x}, U_{t t}, U_{t x}, \ldots\right)=0$,

Introduction a complete variation defines as

$\eta=k x+\omega t, U=U(\eta)$,

And therefore, the eq. 1 construct of ODE of form

$N\left(U, k U^{\prime}, k^{2} U^{\prime \prime}, k^{2} \omega^{2} U^{\prime \prime},-k^{2} \omega U^{\prime \prime}, \ldots\right)=0$,

And then solution of $U(\eta)$ is form of

$$
U(\eta)=\frac{\sum_{n=-c}^{d} a_{n} \exp (n \eta)}{\sum_{m=-p}^{q} b_{m} \exp (m \eta)}=\frac{a_{c} \exp (c \eta)+\ldots+a_{-d} \exp (-d \eta)}{a_{p} \exp (p \eta)+\ldots+a_{-q} \exp (-q \eta)}
$$

Where $c, d, p$ and $q$ are positive integers which are unknown to be further determined, $a_{n}$ and $b_{n}$ are unknown constants.

\section{Application of exp-function method}

\section{The kaup-kupershmidt equation:}

To illustrate the basic idea of the exp-function method, we first consider the kaup- kupershmidt (Parker, 2000) equation in the form

$U_{x x x x x}+U_{t}+45 U_{x} U^{2}-\frac{75}{2} U_{x} U_{x x}-15 U U_{x x x}=0$,

Introducing a complex variation $\eta$ defined as eq. (2), and then eq. (5) becomes an ordinary differential equation, which form of

$$
\omega U^{\prime}+45 k U^{2} U^{\prime}-\frac{75}{2} k^{3} U^{\prime} U^{\prime \prime}-15 k^{3} U U^{\prime \prime \prime}+k^{5} U^{\prime \prime \prime \prime}=0
$$

In order to determine values of $c$ and $p$, we balance the linear term of the highest order $U^{\text {"'" }}$ with the highest order non-linear term $U^{\prime \prime \prime} U$ in eq. (6), we have

$$
\begin{gathered}
U^{\prime \prime \prime \prime}=\frac{c_{1} \exp [(31 p+c) \eta]+\ldots}{c_{2} \exp [32 p \eta]+\ldots}, \\
U U^{\prime \prime}=\frac{c_{3} \exp [(c+7 p) \eta]+\ldots}{c_{4} \exp [8 p \eta]+\ldots} \times \frac{\exp [c \eta]}{\exp [p \eta]}=\frac{c_{3} \exp [(2 c+7 p) \eta]+\ldots}{c_{4} \exp [9 p \eta]+\ldots} \times \frac{\exp [23 p \eta]}{\exp [23 p \eta]},
\end{gathered}
$$

(8)

Where $c_{i}$ are determined coefficients only for simplicity. Balancing highest order of exp-function in eq. (7) and (8), we have

$$
30 p+2 c=c+31 p
$$

Research article

CIndian Society for Education and Environment (iSee)
Goodarziah et al. Indian J.Sci.Technol. 
This leads to the result

$p=c$

Similarly to determine values of $d$ and $q$, we balance the linear term of lowest order in eq. (6)

$U^{\prime \prime \prime \prime}=\frac{\ldots+d_{1} \exp [-(31 q+d) \eta]}{\ldots+d_{2} \exp [-32 q \eta]}$

And

$U^{\prime \prime \prime} U=\frac{\ldots+d_{3} \exp [-(d+7 q) \eta]}{\ldots+d_{4} \exp [-8 q \eta]} \times \frac{\exp [-d \eta]}{\exp [-q \eta]}=\frac{\ldots+d_{3} \exp [-(2 d+7 q) \eta]}{\ldots+d_{4} \exp [-9 q \eta]} \times \frac{\exp [-23 q \eta]}{\exp [-23 q \eta]}$,

Where $d_{i}$ are determined coefficients only for simplicity. Balancing lowest order of exp-function in eq. (11) and (12), we have

$30 q+2 d=d+31 q$

This leads to the result

$q=d$

For simplicity, we set and $p=c=1, d=q=1$ then eq. (4) leads to

$U(\eta)=\frac{a_{1} \exp (\eta)+a_{0}+a_{-1} \exp (-\eta)}{\exp (\eta)+b_{0}+b_{-1} \exp (-\eta)}$

Substituting eq. (15) in to eq. (6), and by the help of MAPLE, we have

$\frac{1}{A}\left\{C_{5} \exp (5 \eta)+C_{4} \exp (4 \eta)+C_{3} \exp (3 \eta)+C_{2} \exp (2 \eta)+C_{1} \exp (\eta)+C_{0}+C_{-1} \exp (-\eta)\right.$
$\left.+C_{-2} \exp (-2 \eta)+C_{-3} \exp (-3 \eta)+C_{-4} \exp (-4 \eta)+C_{-5} \exp (-5 \eta)\right\}=0$,

Where we have

$\mathrm{A}=\left(\left(\exp (\eta)+b_{0}+b_{-1} \exp (-\eta)\right)^{6}\right.$,

and $C_{n}$ are coefficients of $\exp (n \eta)$.equating to zero the coefficients of all powers of $\exp (n \eta)$ yields a set of algebraic equations for $a_{0}, b_{0}, a_{1}, a_{-1}, b_{-1}, \mathrm{k}$ and $\omega$.Solving the system of algebraic equations with the aid of Maple,

We obtain:

Case 1

$b_{-1}=\frac{1}{4} b_{0}^{2}, \quad k=k, \omega=-11 k^{5}, a_{1}=\frac{2}{3} k^{2}, a_{-1}=\frac{1}{6} k^{2} b_{0}^{2}$

$b_{0}=b_{0}, a_{0}=-\frac{10}{3} k^{2} b_{0}$

Case 2

$b_{-1}=\frac{1}{4} b_{0}^{2}, k=k, \omega=-\frac{1}{16} k^{5}, a_{1}=\frac{1}{12} k^{2}, a_{-1}=\frac{1}{48} k^{2} b_{0}^{2}$

$b_{0}=b_{0}, a_{0}=-\frac{5}{12} k^{2} b_{0}$

For case 1

Inserting eq. (18) into (15), one admits to the generalized solitary wave equation of eq. (5) as follows

$U(x, t)=\frac{\frac{2}{3} k^{2} \exp \left(k x-11 k^{5} t\right)-\frac{10}{3} k^{2} b_{0}+\frac{1}{6} k^{2} b_{0}^{2} \exp \left(-\left(k x-11 k^{5} t\right)\right)}{\exp \left(k x-11 k^{5} t\right)+b_{0}+\frac{1}{4} b_{0}^{2} \exp \left(-\left(k x-11 k^{5} t\right)\right)}=\frac{2}{3} k^{2}-\frac{16 b_{0} k^{2}}{4 \exp \left(k x-11 k^{5} t\right)+4 b_{0}+b_{0}^{2} \exp \left(-\left(k x-11 k^{5} t\right)\right)}$ 
In case $k$ and $\omega$ are imaginary numbers, the obtained solitary solution (20) reduces to the periodic solution.

We write $k=\mathrm{iK}$ and using the transformation

$\exp \left(k \mathrm{x}-11 k^{5} \mathrm{t}\right)=\cos \left[K \mathrm{x}-11 K^{5} \mathrm{t}\right]+\mathrm{i} \sin \left[K \mathrm{x}-11 K^{5} \mathrm{t}\right]$

$\exp \left(-\left[k \mathrm{x}-11 k^{5} \mathrm{t}\right]\right)=\cos \left[K \mathrm{x}-11 K^{5} \mathrm{t}\right]-\mathrm{i} \sin \left[K \mathrm{x}-11 K^{5} \mathrm{t}\right]$

Substituting eq. (21) into (20) results in a periodic solution

$$
U=-\frac{2}{3} K^{2}+\frac{16 K^{2} b_{0}}{\left(4+b_{0}^{2}\right) \cos \left(K x-11 K^{5} t\right)+4 b_{0}+\left(4-b_{0}^{2}\right) i \sin \left(K x-11 K^{5} t\right)}
$$

If we search for a periodic solution or compact-like solution, the imaginary part in eq. (22) must be zero that requires that

$4-\mathrm{b}_{0}^{2}=0$.

From eq. (23) we obtain

$\mathrm{b}_{0}= \pm 2$.

Substituting $\mathrm{b}_{0}=2$ into eq. (22) results

$U_{1}(x, t)=\frac{32 K^{2}}{8 \cos \left(K x-11 K^{5} t\right)+8}-\frac{2}{3} K^{2}=\frac{2 K^{2}}{\cos ^{2}\left(\frac{K}{2} x-\frac{11}{2} K^{5} t\right)}-\frac{2}{3} K^{2}$

And substituting $\mathrm{b}_{0}=-2$ into eq. (22) results

$U_{2}(x, t)=\frac{-32 K^{2}}{8 \cos \left(K x-11 K^{5} t\right)-8}-\frac{2}{3} K^{2}=\frac{2 K^{2}}{\sin ^{2}\left(\frac{K}{2} x-\frac{11}{2} K^{5} t\right)}-\frac{2}{3} K^{2}$

Whereas $k=i \mathrm{~K}$, we write $\mathrm{K}=-i k$ and using the transformation into eq. (25), (26)

$$
\begin{aligned}
& U_{3}(x, t)=-\frac{2 k^{2}}{\cos ^{2}\left[i\left(\frac{1}{2} k x-\frac{11}{2} k^{5} t\right)\right]}+\frac{2}{3} k^{2}=-2 k^{2} \operatorname{sech}^{2}\left(\frac{k}{2} x-\frac{11}{2} k^{5} t\right)+\frac{2}{3} k^{2} \\
& U_{4}(x, t)=-\frac{2 k^{2}}{\sin ^{2}\left[i\left(\frac{1}{2} k x-\frac{11}{2} k^{5} t\right)\right]}+\frac{2}{3} k^{2}=2 k^{2} \operatorname{csch}^{2}\left(\frac{k}{2} x-\frac{11}{2} k^{5} t\right)+\frac{2}{3} k^{2}
\end{aligned}
$$

For case 2, inserting eq. (19) into (15), one admits to the generalized solitary wave equation of eq. (5) as follows

$U(x, t)=\frac{\frac{1}{12} k^{2} \exp \left(k x-\frac{1}{16} k^{5} t\right)-\frac{5}{12} k^{2} b_{0}+\frac{1}{48} k^{2} b_{0}^{2} \exp \left(-\left(k x-\frac{1}{16} k^{5} t\right)\right)}{\exp \left(k x-\frac{1}{16} k^{5} t\right)+b_{0}+\frac{1}{4} b_{0}^{2} \exp \left(-\left(k x-\frac{1}{16} k^{5} t\right)\right)}=\frac{1}{12} k^{2}-\frac{2 k^{2} b_{0}}{4 \exp \left(k x-\frac{1}{16} k^{5} t\right)+4 b_{0}+b_{0}^{2} \exp \left(-\left(k x-\frac{1}{16} k^{5} t\right)\right)}$

In case $k$ and $\omega$ are imaginary numbers, the obtained solitary solution (29) reduces to the periodic solution.

We write $k=\mathrm{iK}$ and using the transformation

$$
\begin{aligned}
& \exp \left(k x-\frac{1}{16} k^{5} \mathrm{t}\right)=\cos \left[K \mathrm{x}-\frac{1}{16} K^{5} \mathrm{t}\right]+\mathrm{i} \sin \left[K \mathrm{x}-\frac{1}{16} K^{5} \mathrm{t}\right] \\
& \exp \left(-\left[k \mathrm{x}-\frac{1}{16} k^{5} \mathrm{t}\right]\right)=\cos \left[K \mathrm{x}-\frac{1}{16} K^{5} \mathrm{t}\right]-\mathrm{i} \sin \left[K \mathrm{x}-\frac{1}{16} K^{5} \mathrm{t}\right]
\end{aligned}
$$

Substituting eq. (30) into (29) results in a periodic solution

$$
U=-\frac{1}{12} K^{2}+\frac{2 K^{2} b_{0}}{\left(4+b_{0}^{2}\right) \cos \left(K x-\frac{1}{16} K^{5} t\right)+4 b_{0}+\left(4-b_{0}^{2}\right) i \sin \left(K x-\frac{1}{16} K^{5} t\right)}
$$


Indian Journal of Science and Technology

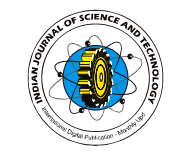

If we search for a periodic solution or compact-like solution, the imaginary part in eq. (31) must be zero, substituting $b_{0}=2$ into eq. (31) results

$$
U_{5}(x, t)=\frac{4 K^{2}}{8 \cos \left(K x-\frac{1}{16} K^{5} t\right)+8}-\frac{1}{12} K^{2}=\frac{K^{2}}{4 \cos ^{2}\left(\frac{K}{2} x-\frac{1}{32} K^{5} t\right)}-\frac{1}{12} K^{2}
$$

And substituting $b_{0}=-2$ into eq. (31) results

$$
U_{6}(x, t)=\frac{-4 K^{2}}{8 \cos \left(K x-\frac{1}{16} K^{5} t\right)-8}-\frac{1}{12} K^{2}=\frac{K^{2}}{4 \sin ^{2}\left(\frac{K}{2} x-\frac{1}{32} K^{5} t\right)}-\frac{1}{12} K^{2}
$$

Whereas $k=\mathrm{iK}$, we write $\mathrm{K}=-i k$ and using the transformation into eq. (32), (33)

$$
\begin{aligned}
& U_{7}(x, t)=-\frac{k^{2}}{4 \cos ^{2}\left[i\left(\frac{1}{2} k x-\frac{1}{32} k^{5} t\right)\right]}+\frac{1}{12} k^{2}=-\frac{1}{4} k^{2} \operatorname{sech}^{2}\left(\frac{k}{2} x-\frac{1}{32} k^{5} t\right)+\frac{1}{12} k^{2} \\
& U_{8}(x, t)=-\frac{k^{2}}{4 \sin ^{2}\left[i\left(\frac{1}{2} k x-\frac{1}{32} k^{5} t\right)\right]}+\frac{1}{12} k^{2}=\frac{1}{4} k^{2} \operatorname{csch}^{2}\left(\frac{k}{2} x-\frac{1}{32} k^{5} t\right)+\frac{1}{12} k^{2}
\end{aligned}
$$

(2+1)-dimensional potential Kadomtsev-Petviashvili (PKP) equation

Now, let us consider the PKP eq. [15] in the form

$\frac{1}{4} U_{x x x x}+\frac{3}{2} U_{x} U_{x x}+\frac{3}{4} U_{y y}+U_{x t}=0$,

Using the transformation

$\eta=k x+l y+\omega t \quad, U=U(\eta)$

Then eq. (37) becomes an ordinary differential equation, which form of

$\mathrm{U}(\eta)=\frac{1}{4} k^{4} U^{\prime \prime \prime}+\frac{3}{2} k^{3} U^{\prime} U^{\prime \prime}+\left(\frac{3}{4} l^{2}+k \omega\right) U^{\prime \prime}=0$,

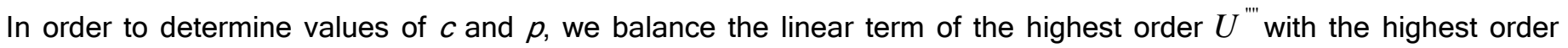
non-linear term $U^{\prime} U^{\prime \prime}$ in eq. (38), we have

$$
\begin{aligned}
& U^{\prime \prime \prime}=\frac{c_{1} \exp [(15 p+c) \eta]+\ldots}{c_{2} \exp [16 p \eta]+\ldots}, \\
& U^{\prime} U^{\prime \prime}=\frac{c_{3} \exp [(c+3 p) \eta]+\ldots}{c_{4} \exp [4 p \eta]+\ldots} \times \frac{\exp [(c+p) \eta]}{\exp [2 p \eta]}=\frac{c_{3} \exp [(2 c+4 p) \eta]+\ldots}{c_{4} \exp [6 p \eta]+\ldots} \times \frac{\exp [10 p \eta]}{\exp [10 p \eta]}
\end{aligned}
$$

Where $c_{i}$ are determined coefficients only for simplicity. Balancing highest order of exp function in eq. (39) and (40), we have

$15 p+c=2 c+14 p$

This leads to the result

$p=c$

Similarly to determine values of $d$ and $q$, we balance the linear term of lowest order in Eq.(38)

$U^{\prime \prime \prime}=\frac{\ldots+d_{1} \exp [-(15 q+d) \eta]}{\ldots+d_{2} \exp [-16 q \eta]}$

and 
Indian Journal of Science and Technology

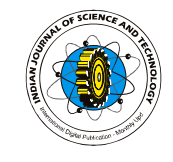

$$
U^{\prime} U^{\prime \prime}=\frac{\ldots+d_{3} \exp [-(d+3 q) \eta]}{\ldots+d_{4} \exp [-4 q \eta]} \times \frac{\exp [-(d+q) \eta]}{\exp [-2 q \eta]}=\frac{\ldots+d_{3} \exp [-(2 d+4 q) \eta]}{\ldots+d_{4} \exp [-6 q \eta]} \times \frac{\exp [-10 q \eta]}{\exp [-10 q \eta]}
$$

ISSN: 0974- 6846

Vol. 4 No. 2 (Feb 2011)

Where $d_{i}$ are determined coefficients only for simplicity. Balancing lowest order of exp-function in eq. (43) and (44), we have

$15 q+d=2 d+14 q$

This leads to the result

$q=d$

For simplicity, we set and $p=c=1, d=q=1$ then eq. (4) leads to

$U(\eta)=\frac{a_{1} \exp (\eta)+a_{0}+a_{-1} \exp (-\eta)}{\exp (\eta)+b_{0}+b_{-1} \exp (-\eta)}$

Substituting eq. (47) in to eq. (38), and by the help of MAPLE, we have

$$
\begin{aligned}
& \frac{1}{A}\left\{C_{4} \exp (4 \eta)+C_{3} \exp (3 \eta)+C_{2} \exp (2 \eta)+C_{1} \exp (\eta)+C_{0}+C_{-1} \exp (-\eta)\right. \\
& \left.+C_{-2} \exp (-2 \eta)+C_{-3} \exp (-3 \eta)+C_{-4} \exp (-4 \eta)\right\}=0
\end{aligned}
$$

Where we have

$\mathrm{A}=\left(\left(\exp (\eta)+b_{0}+b_{-1} \exp (-\eta)\right)^{5}\right.$

and $C_{n}$ are coefficients of $\exp (n \eta)$. equating to zero the coefficients of all powers of $\exp (n \eta)$ yields a set of algebraic equations for $a_{0}, b_{0}, a_{1}, a_{-1}, b_{-1}, \mathrm{k}, l$ and $\omega$. Solving the system of algebraic equations with the aid of Maple, we obtain :

$\omega=-\frac{3 l^{2}+k^{4}}{4 k}, a_{1}=a_{1}, a_{-1}=-\frac{4 a_{1} b_{0}^{2} k^{2}-4 a_{0} b_{0} k^{2}-4 a_{1}^{2} b_{0}^{2} k+6 a_{1} b_{0} a_{0} k+a_{1} a_{0}^{2}+a_{1}^{3} b_{0}^{2}-2 a_{1}^{2} b_{0} a_{0}-2 a_{0}^{2} k}{4 k^{2}}$

$b_{0}=b_{0}, a_{0}=a_{0}, b_{-1}=-\frac{-2 k a_{1} b_{0}^{2}+2 k a_{0} b_{0}+a_{0}^{2}+a_{1}^{2} b_{0}^{2}-2 a_{1} b_{0} a_{0}}{4 k^{2}}, k=k, l=l$,

Inserting eq. (50) into (47), admits to the generalized solitary wave equation of eq. (36) as follows

$$
\begin{aligned}
& U(x, y, z, t)=\frac{a_{1} \exp \left(k x+l y-\frac{k^{4}+3 l^{2}}{4 k} t\right)+a_{0}-\frac{4 a_{1} b_{0}^{2} k^{2}-4 a_{0} b_{0} k^{2}-4 a_{1}^{2} b_{0}^{2} k+6 a_{1} a_{0} b_{0} k+a_{1} a_{0}^{2}+a_{1}^{3} b_{0}^{2}-2 a_{0} b_{0} a_{1}^{2}-2 k a_{0}^{2}}{4 k^{2}} \exp \left(-\left(k x+l y-\frac{k^{4}+3 l^{2}}{4 k} t\right)\right)}{\exp \left(k x+l y-\frac{k^{4}+3 l^{2}}{4 k} t\right)+b_{0}+\frac{2 a_{1} b_{0}^{2} k-2 a_{0} b_{0} k-a_{0}^{2}-a_{1}^{2} b_{0}^{2}+2 a_{1} b_{0} a_{0}}{4 k^{2}} \exp \left(-\left(k x+l y-\frac{k^{4}+3 l^{2}}{4 k} t\right)\right)}= \\
& a_{1}-\frac{4 k^{2} a_{1} b_{0}-4 k^{2} a_{0}+2 k\left(2 a_{1} b_{0}^{2} k-2 a_{0} b_{0} k-a_{1}^{2} b_{0}^{2}+2 a_{1} a_{0} b_{0}-a_{0}^{2}\right) \exp \left(-\left(k x+l y-\frac{k^{4}+3 l^{2}}{4 k} t\right)\right)}{4 k^{2} \exp \left(k x+l y-\frac{k^{4}+3 l^{2}}{4 k} t\right)+4 b_{0} k^{2}+\left(2 k a_{1} b_{0}^{2}-2 k a_{0} b_{0}-a_{0}^{2}-a_{1}^{2} b_{0}^{2}+2 a_{1} a_{0} b_{0}\right) \exp \left(-\left(k x+l y-\frac{k^{4}+3 l^{2}}{4 k} t\right)\right)}
\end{aligned}
$$

In case $\mathrm{k}, l$ and $\omega$ are imaginary numbers, the obtained solitary solution (51) reduces to the periodic solution. We write $\mathrm{k}=\mathrm{iK}, l=\mathrm{iL}$ and using the transformation

$$
\begin{aligned}
& \exp \left(k x+l y-\frac{3 l^{2}+k^{4}}{4 k} t\right)=\cos \left[K x+L y+\frac{K^{4}-3 L^{2}}{4 K} t\right]+\mathrm{i} \sin \left[K x+L y+\frac{K^{4}-3 L^{2}}{4 K} t\right], \\
& \exp \left(-\left[k x+l y-\frac{3 l^{2}+k^{4}}{4 k} t\right]\right)=\cos \left[K x+L y+\frac{K^{4}-3 L^{2}}{4 K} t\right]-i \sin \left[K x+L y+\frac{K^{4}-3 L^{2}}{4 K} t\right],
\end{aligned}
$$

Substituting eq. (52) into (51) results in a periodic solution

$$
U(x, y, t)=a_{1}+\frac{2 K\left[-2 a_{0} K+2 a_{1} b_{0} K+\left(2 K a_{1} b_{0}^{2}-2 K a_{0} b_{0}\right) \cos (\eta)+\left(a_{1}^{2} b_{0}^{2}+a_{0}^{2}-2 a_{1} a_{0} b_{0}\right) \sin (\eta)+\left(a_{1}^{2} b_{0}^{2}+a_{0}^{2}-2 a_{1} a_{0} b_{0}\right) i \cos (\eta)+\left(-2 K a_{1} b_{0}^{2}+2 K a_{0} b_{0}\right) i \sin (\eta)\right]}{\left(-4 K^{2}-a_{0}^{2}-a_{1}^{2} b_{0}^{2}+2 a_{1} a_{0} b_{0}\right) \cos (\eta)-4 b_{0} K^{2}+\left(2 K a_{1} b_{0}^{2}-2 K a_{0} b_{0}\right) \sin (\eta)+\left(2 K a_{1} b_{0}^{2}-2 K a_{0} b_{0}\right) i \cos (\eta)+\left(-4 K^{2}+a_{0}^{2}+a_{1}^{2} b_{0}^{2}-2 a_{1} a_{0} b_{0}\right) i \sin (\eta)}
$$


Where in this case $\eta=K x+L y+\frac{K^{4}-3 L^{2}}{4 K} t$ and $a_{1}, a_{0}$ and $b_{0}$ are free parameters. If we

set $b_{0}=0, a_{0}= \pm 2 K$ in eq. (53) becomes

$\mathrm{U}(\mathrm{x}, \mathrm{y}, \mathrm{t})=\mathrm{a}_{1}-K i \pm K \sec \left(K x+L y+\frac{K^{4}-3 L^{2}}{4 K} t\right)-K \tan \left(K x+L y+\frac{K^{4}-3 L^{2}}{4 K} t\right)$

Whereas $\mathrm{k}=\mathrm{iK}, l=\mathrm{iL}$, we write $\mathrm{K}=-i k, \mathrm{~L}=-i l$ and with Substituting into (54) we obtain

$$
\left.\mathrm{U}(\mathrm{x}, \mathrm{y}, \mathrm{t})=\mathrm{a}_{1}-k \pm k i \operatorname{sech}\left(k x+l y-\frac{3 l^{2}+k^{4}}{4 k} t\right)+k \tanh \left(k x+l y-\frac{3 l^{2}+k^{4}}{4 k} t\right)\right]
$$

\section{Conclusion}

It can be concluded that the exp-function method is very powerful and efficient technique in finding exact solutions for wide classes of problems. The exp-function method has got many merits and much more advantages than the exact solutions. Calculations in the exp-function method are simple and straightforward. The reliability of the method and the reduction in the size of computational domain give this method a wider applicability. The results show that the exp-function method is a powerful mathematical tool for solving systems of non-linear partial differential equations having wide applications in engineering.

\section{References}

1. Burden RL and Faires JD (1993) Numerical analysis. PWS publishing company, $5^{\text {th }}$ edn., Boston.

2. Gan Kaya D and El-Sayed M (2003) Numerical soliton-like solutions of the potential KadomtsevPetviashvili equation by the decomposition method. Phy. Letts. A. 320, 192-199.

3. He (2004) Comparison of homotopy perturbation method and homotopy analysis method. Appl. Math. Computat. 156, 527-539.

4. He (200b) A coupling method of a homotopy technique and a perturbation technique for non-linear problems. Int. J. Non-Linear Mech. 35, 37-43.

5. He JH (2000a) Variational iteration method for autonomous ordinary differential systems. Appl. Math. Computat. 114, 115-123.

6. He JH (2004) Homotopy perturbation method: A new non-linear analytical technique. Appl. Math. Computat. 156, 591-596.

7. He JH (2005) Application of homotopy perturbation method to non-linear wave equations. Chaos Solitons Fractals. 26, 695-700.

8. He JH (2006) Exp-function method for non-linear wave equations. Chaos Solitons Fractals. 30, 700-708.

9. He JH (2006a) Non-perturbative method for strongly non-linear problems. Berlin: dissertation. De-Verlag im internet $\mathrm{GmbH}$.

10. He JH and Abdou MA (2007) New periodic solutions for non-linear evolution equations using exp-function method. Chaos Solitons Fractals. 34, 1421-1429.
11.Kaya D and El-Sayed SM (2003) On a generalized fifth order KdV equations. Phy. Letts. A. 310, 44-51.

12.Parker A (2000) On soliton solutions of the KaupKupershmidt equation. I. Direct bilinearisation and solitary wave. Physica D. 137, 25-33.

13.Zhang SD (2007) Application of exp-function method to a KdV equation with variable coefficients. Phy. Letts. A. 365, 448-453.

14.Zhu SD (2007a) Exp-function method for the hybridlattice system. Int. J. Non-linear Sci. Numerical Soln. 8(3), 461-464.

15.Zhu SD (2007b) Exp-function method for the discrete mKdV lattice. Int. J. Non-linear Sci. Numerical Soln. 8(3), 465-468.
Goodarziah et al. Indian J.Sci.Technol. 